\title{
Deterministic Limit of Intracellular Calcium Spikes
}

\author{
V. Voorsluijs, ${ }^{1, *}$ S. Ponce Dawson, ${ }^{2}$ Y. De Decker, ${ }^{1}$ and G. Dupont ${ }^{3}$ \\ ${ }^{1}$ Nonlinear Physical Chemistry Unit and Center for Nonlinear Phenomena and Complex Systems (CENOLI), \\ Université libre de Bruxelles, Boulevard du Triomphe, C.P. 231, B-1050 Brussels, Belgium \\ ${ }^{2}$ Departamento de Física, FCEN-UBA and IFIBA, UBA-CONICET, Ciudad Universitaria, \\ Pabellón I, 1428 Buenos Aires, Argentina \\ ${ }^{3}$ Unité de Chronobiologie Théorique, Université libre de Bruxelles, \\ Boulevard du Triomphe, C.P. 231, B-1050 Brussels, Belgium
}

(Received 10 October 2018; revised manuscript received 9 January 2019; published 26 February 2019)

\begin{abstract}
In nonexcitable cells, global $\mathrm{Ca}^{2+}$ spikes emerge from the collective dynamics of clusters of $\mathrm{Ca}^{2+}$ channels that are coupled by diffusion. Current modeling approaches have opposed stochastic descriptions of these systems to purely deterministic models, while both paradoxically appear compatible with experimental data. Combining fully stochastic simulations and mean-field analyses, we demonstrate that these two approaches can be reconciled. Our fully stochastic model generates spike sequences that can be seen as noise-perturbed oscillations of deterministic origin, while displaying statistical properties in agreement with experimental data. These underlying deterministic oscillations arise from a phenomenological spike nucleation mechanism.
\end{abstract}

DOI: 10.1103/PhysRevLett.122.088101

The intracellular calcium ion $\mathrm{Ca}^{2+}$ is a major second messenger involved in many signaling pathways [1]. On average, the cell tends to maintain its cytosolic $\mathrm{Ca}^{2+}$ concentration $\left[\mathrm{Ca}^{2+}\right]$ to a low basal value of about $100 \mathrm{nM}$, but transient rises of $\left[\mathrm{Ca}^{2+}\right]$ can occur upon stimulation of the cell by an external agonist. The frequency and amplitude of these signals encode information about the nature and intensity of the physiological response [1].

In this Letter, we focus on variations of $\left[\mathrm{Ca}^{2+}\right]$ initiated by an increase in the concentration of inositol 1,4,5trisphosphate $\left(\mathrm{IP}_{3}\right)$ and involving $\mathrm{Ca}^{2+}$ exchanges between the cytosol and the endoplasmic reticulum (ER). These exchanges are ensured by dedicated pumps and channels located in the membrane of the ER. The ER is able to sequestrate $\mathrm{Ca}^{2+}$ via endoplasmic reticulum calcium adenosine triphosphatases (SERCA pumps), while $\mathrm{IP}_{3}$ receptor $\left(\mathrm{IP}_{3} \mathrm{R}\right)$ channels enable $\mathrm{Ca}^{2+}$ release from the ER to the cytosol. $\mathrm{IP}_{3} \mathrm{Rs}$ are activated at low $\left[\mathrm{Ca}^{2+}\right]$ (resulting in $\mathrm{Ca}^{2+}$-induced $\mathrm{Ca}^{2+}$ release, or CICR), but are inhibited at high $\left[\mathrm{Ca}^{2+}\right][2] . \mathrm{IP}_{3} \mathrm{Rs}$ typically form multichannel clusters of 300-800 $\mathrm{nm}$ in width [3,4], separated by distances ranging from 1 to $7 \mu \mathrm{m}[5,6]$.

The combination of CICR and $\mathrm{Ca}^{2+}$ diffusion leads to complex collective behaviors at different scales. The concerted release of $\mathrm{Ca}^{2+}$ through several channels in a single cluster can lead to a local rapid increase of $\left[\mathrm{Ca}^{2+}\right]$, which is known as a "puff." Such subcellular $\mathrm{Ca}^{2+}$ signals occur randomly, last less than $1 \mathrm{~s}$, and have a spatial extent of a few microns [3-6]. At the cell level, the coupling of clusters can result in the emergence of global $\left[\mathrm{Ca}^{2+}\right]$ increases, invading the whole cell and lasting at least a few seconds. These global $\mathrm{Ca}^{2+}$ events are called "spikes" $[7,8]$. While the mean interpuff time interval (IPI) usually does not exceed $5 \mathrm{~s}$, the interspike time interval (ISI) ranges between $20 \mathrm{~s}$ and several minutes [5,8]. This difference points to the existence of different types of dynamics for the termination of spikes and puffs.

The statistical distributions of IPIs and ISIs are different. On one hand, IPIs are essentially random events and obey Poisson distributions with a mean mainly determined by the inhibition and reactivation timescales of $\mathrm{IP}_{3} \mathrm{Rs}$ [9]. The dynamics of $\mathrm{IP}_{3}$ Rs clusters is thus intrinsically stochastic and aperiodic $[8,10]$. On the other hand, ISIs generally include a nonstochastic, absolute refractory period $\left(T_{\min }\right)$ that cannot exclusively be accounted for by the inhibition and recovery of channels alone and more likely results from the existence of a deterministic global process related to $\mathrm{IP}_{3}$ metabolism [11,12]. ISIs can be significantly larger than $T_{\min }$, which would not be the case if spikes were purely deterministic processes. ISIs are thus often divided into deterministic and stochastic $\left(T_{\text {stoch }}\right)$ contributions, ISI $=T_{\min }+T_{\text {stoch }}$. Experiments show that $T_{\text {stoch }}$ creates a linear relation between the standard deviation of the ISI $\left(\sigma_{\mathrm{ISI}}\right)$ and their mean $\left(T_{\mathrm{av}}\right)$,

$$
\sigma_{\mathrm{ISI}}=\alpha\left(T_{\mathrm{av}}-T_{\min }\right),
$$

where the slope $\alpha$ varies according to the cell type and the agonist used to induce spikes $[11,13]$.

Cell-level spikes can be surprisingly well described either by deterministic models with oscillatory dynamics 


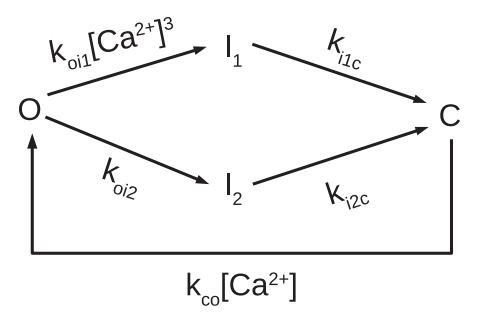

FIG. 1. Model describing the dynamics of a cluster of $\mathrm{IP}_{3} \mathrm{Rs}$ from Calabrese et al. [22].

[14] or by stochastic models, based on the coupling of clusters whose local dynamics is aperiodic [15] or on intensity functions describing spikes from a top-down perspective [16]. Experiments [5] strongly suggest that global spikes and waves emerge from a nucleation mechanism, which is reproduced by deterministic models considering excitable clusters [17]. There is thus no consensus about the nonlinear behavior underlying global spikes [18]. In this Letter, we propose a unifying heuristic model, based on a nucleation mechanism. Combining fully stochastic simulations and bifurcation analyses of this model, we generated spike sequences corresponding to oscillations in the mean-field limit and displaying statistical properties compatible with experimental data.

The cell is modeled as a two-dimensional system with periodic boundary conditions, representing a layer of cytosol above the surface of the ER. The system is discretized in $N_{\text {tot }}$ square compartments of side length $\Delta x$ and volume $V_{c}$, where the $\left[\mathrm{Ca}^{2+}\right]$ is considered to be homogeneous, and some of these compartments contain clusters of $\mathrm{IP}_{3} \mathrm{Rs}$. The stochastic behavior of individual channels and the associated steep $\mathrm{Ca}^{2+}$ gradients were explicitly described in many previous models, but such simulations were computationally expensive and called for approximations [19-21]. Rather than simulate individual $\mathrm{IP}_{3} \mathrm{Rs}$, we based our approach on an extension of the phenomenological cluster model (Fig. 1), developed by Calabrese et al. [22] and validated against experimental observations.

Each cluster as a whole can be in four different states: an open state $O$, a closed state $C$, or one of the two inhibited states $I_{1}$ and $I_{2}$, and transitions between these states take place according to the scheme shown in Fig. $1 . \mathrm{Ca}^{2+}$ release exclusively occurs when the cluster is in state $O$. In order to model the CICR mechanism, the transition probability from $C$ to $O$ depends on $\left[\mathrm{Ca}^{2+}\right]$, which ensures that the probability to trigger cluster activity increases with the cytosolic $\mathrm{Ca}^{2+}$ content [23]. When the cluster is in state $O$, two different pathways are available. The cluster can become rapidly inhibited to reach state $I_{2}$. The characteristic time of the $I_{2} \rightarrow C$ transition, $1 / k_{i 2 c} \approx 0.5 \mathrm{~s}$, is compatible with the recovery time of a cluster after a puff. Once in state $I_{2}$, the cluster can recover and come back to state $C$. At high ambient $\left[\mathrm{Ca}^{2+}\right]$ - and hence very high local $\left[\mathrm{Ca}^{2+}\right]$ - the cluster can also become inhibited in state $I_{1}$,
TABLE I. Simulations parameters.

\begin{tabular}{lll}
\hline \hline$k_{c o}$ & {$[5-20] \mu \mathrm{M}^{-1} \mathrm{~s}^{-1}$} & $C \rightarrow O$ \\
$k_{o i 1}$ & $0.05 \mu \mathrm{M}^{-3} \mathrm{~s}^{-1}$ & $O \rightarrow I_{1}$ \\
$k_{o i 2}$ & $40 \mathrm{~s}^{-1}$ & $O \rightarrow I_{2}$ \\
$k_{i 1 c}$ & $0.005 \mathrm{~s}^{-1}$ & $I_{1} \rightarrow C$ \\
$k_{i 2 c}$ & $2 \mathrm{~s}^{-1}$ & $I_{2} \rightarrow C$ \\
$v_{p}$ & {$[0.7-0.9] \mu \mathrm{M} \mathrm{s}^{-1}$} & Maximal rate of SERCA \\
$K_{p}$ & $0.1 \mu \mathrm{M}$ & SERCA binding constant \\
{$\left[\mathrm{Ca}^{2+}\right]_{b}$} & $0.04 \mu \mathrm{M}$ & Basal $\left[\mathrm{Ca}^{2+}\right]$ \\
$D$ & $40 \mu \mathrm{m}^{2} \mathrm{~s}^{-1}$ & Ca ${ }^{2+}$ diffusion coefficient \\
$\Delta x$ & $0.5 \mu \mathrm{m}$ & Length of a compartment \\
$V_{c}$ & $10^{-16} \mathrm{~L}$ & Volume of a compartment \\
\hline \hline
\end{tabular}

from which recovery is slower than from $I_{2}$. This assumption is based on the observation [5] that when puffs have contributed to the onset of a global $\mathrm{Ca}^{2+}$ spike, puff activity is reduced. In the model, the dependence on $\left[\mathrm{Ca}^{2+}\right]^{3}$ in the probability of $O \rightarrow I_{1}$ is hence combined to a long recovery time (see Table I).

Considering that open clusters release $\mathrm{Ca}^{2+}$ at a constant rate $\Sigma$ [24], the amount of $\mathrm{Ca}^{2+}$ released depends on the time spent by clusters in state $O$, which is ruled by the kinetic scheme described above. We evaluate $\Sigma$ as $\Sigma=I_{\text {eff }} \times 10^{-6} / 2 V_{c} F$, where $I_{\text {eff }}=I(1-b)$ is an effective current accounting for the trapping of a fraction of ions by buffers, $F=96485 \mathrm{C} \mathrm{mol}^{-1}$ is the Faraday constant and the factor $10^{-6}$ ensures that $I_{\text {eff }}$ is in picoamperes while $\Sigma$ is in $\mu \mathrm{M} \mathrm{s}^{-1}$. To be consistent with a current $I$ between 0.12 and $0.95 \mathrm{pA}$, as reported by Bruno et al. [20], and for a buffering $b$ of $98 \%$ and $V_{c}=10^{-16} \mathrm{~L}, \Sigma$ ranges from 124 to $984 \mu \mathrm{M} \mathrm{s}^{-1}$ depending on the simulated stimulation level.

Contrary to the majority of previous models for global $\mathrm{Ca}^{2+}$ signals, we simulated the dynamics of the system with a fully stochastic approach based on Gillespie's algorithm [25] (see Supplemental Material [26]). Fluxes associated with leak from the ER and uptake by SERCA pumps are also described as stochastic processes ruled by well-established kinetics, whose associated parameters are also given in Table I. Stochastic diffusion is introduced as a jump process between two neighboring boxes of the system.

We thus included a total of nine different stochastic processes in our description: the five transitions relative to cluster states, $\mathrm{Ca}^{2+}$ fluxes due to leak from the ER and uptake by SERCA pumps in each compartment, $\mathrm{Ca}^{2+}$ diffusion between compartments, and release of $\mathrm{Ca}^{2+}$ from open clusters. The propensity functions associated with these processes are given in the Supplemental Material [26] (Table S1).

Experimentally, puff sequences are recorded in the presence of EGTA, an exogeneous slow buffer that prevents diffusion-mediated coupling between clusters [8]. To mimic 


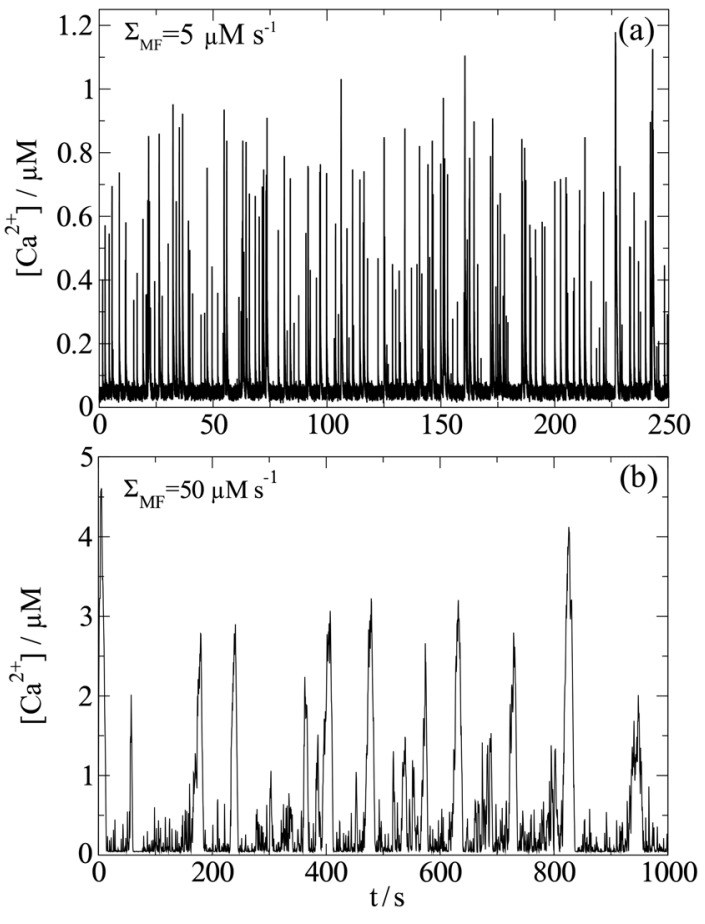

FIG. 2. (a) Puff sequence showing the local $\left[\mathrm{Ca}^{2+}\right]$ averaged in $1 \mathrm{fL}$ around an isolated cluster, corresponding to experimental observations in the presence of EGTA. $\Sigma_{\mathrm{MF}}=5 \mu \mathrm{M} \mathrm{s}^{-1}$ ( $\Sigma=500 \mu \mathrm{M} \mathrm{s}^{-1}$ and $\bar{\rho}_{c}=0.01$ ). (b) Spike sequence showing the global $\left[\mathrm{Ca}^{2+}\right]$ averaged over the whole system, corresponding to experimental observations in the absence of EGTA. $\Sigma_{\mathrm{MF}}=$ $50 \mu \mathrm{M} \mathrm{s}^{-1}\left(\Sigma=500 \mu \mathrm{M} \mathrm{s}^{-1}\right.$ and $\left.\bar{\rho}_{c}=0.1\right)$. The mean ISI is $(102.1 \pm 50.4) \mathrm{s}$. (a),(b) The whole system size is $5 \times 5 \mu \mathrm{m}^{2}$, $k_{c o}=20 \mu \mathrm{M}^{-1} \mathrm{~s}^{-1}, v_{p}=0.9 \mu \mathrm{M} \mathrm{s}^{-1}$, and the other parameters are given in Table I.

such conditions, we considered a $0.5 \times 0.5 \mu \mathrm{m}^{2}$ single cluster located in the middle of a $5 \times 5 \mu \mathrm{m}^{2}$ system. The resulting dynamics is characterized by small amplitude bursts of $\left[\mathrm{Ca}^{2+}\right]$ that resemble the experimentally observed puffs [see Fig. 2(a)]. Puff amplitudes were calculated as the maximal average $\left[\mathrm{Ca}^{2+}\right]$ reached during a puff in a volume of $1 \mathrm{fL}$ around the cluster, i.e., in the compartment containing the cluster and its eight first neighbors [19]. As shown in Table II, amplitude, IPI, and puff duration statistics obtained for $\Sigma=500 \mu \mathrm{M} \mathrm{s}^{-1}$ are consistent with those reported from experiments $[6,9,27]$.

TABLE II. Mean and standard deviation of the $\left[\mathrm{Ca}^{2+}\right]$ amplitude measured in $1 \mathrm{fL}$ around the cluster $(A)$, IPI, and full duration at half maximum amplitude (FDHM).

\begin{tabular}{lccc}
\hline \hline & This work & Experiments & Ref. \\
\hline$A(\mu \mathrm{M})$ & $0.588 \pm 0.211$ & $0.216 \pm 0.04$ & {$[27]$} \\
IPI $(\mathrm{s})$ & $1.94 \pm 1.2$ & $1.50 \pm 1.37$ & {$[9]$} \\
FDHM $(\mathrm{ms})$ & 40 & 45 & {$[6]$} \\
\hline \hline
\end{tabular}

In order to gain insight into the nonlinear dynamics underlying this behavior, we turned to a deterministic version of the model. We considered an ensemble of clusters experiencing the same average $\left[\mathrm{Ca}^{2+}\right]$ and interacting globally via this effective concentration field. With such an approach, the $\mathrm{Ca}^{2+}$ dynamics in the intercluster spaces is not described explicitly, but clusters are assumed to be distributed on the membrane of the ER according to a given cluster density $\bar{\rho}_{c}=N_{c} / N_{\text {tot }}$, where $N_{c}$ is the number of clusters in the system. We considered $\bar{\rho}_{c}=0.1$, which corresponds to an average intercluster distance of $1 \mu \mathrm{m}$ and is consistent with experimental data [6].

Evolution equations for $\left[\mathrm{Ca}^{2+}\right]$ and for the fraction of clusters $x=N_{X} / N_{c}$ in state $X=O, I_{1}, I_{2}$, or $C$ can be easily derived in this mean-field approximation (see Supplemental Material [26]). The deterministic dynamics of the system is ruled by the following evolution equations:

$$
\begin{aligned}
\frac{d o}{d t} & =k_{c o}\left[\mathrm{Ca}^{2+}\right]\left(1-o-i_{1}-i_{2}\right)-k_{o i 1}\left[\mathrm{Ca}^{2+}\right]^{3} o-k_{o i 2} o \\
\frac{d i_{1}}{d t} & =k_{o i 1}\left[\mathrm{Ca}^{2+}\right]^{3} o-k_{i 1 c} i_{1} \\
\frac{d i_{2}}{d t} & =k_{o i 2} o-k_{i 2 c} i_{2} \\
\frac{d\left[\mathrm{Ca}^{2+}\right]}{d t} & =\Sigma_{\mathrm{MF}} o-J_{\mathrm{SERCA}}+J_{\text {leak }}
\end{aligned}
$$

where $\Sigma_{\mathrm{MF}}=\Sigma \bar{\rho}_{c}$ is a rescaled release rate. In these equations, only the fractions $o, i_{1}$, and $i_{2}$ appear because of the conservation relation $o+i_{1}+i_{2}+c=1$. The SERCA and leak fluxes are given by

$$
J_{\text {SERCA }}=\frac{v_{p}\left[\mathrm{Ca}^{2+}\right]^{2}}{\left[\mathrm{Ca}^{2+}\right]^{2}+K_{p}^{2}}
$$

and

$$
J_{\text {leak }}=\frac{v_{p}\left[\mathrm{Ca}^{2+}\right]_{b}^{2}}{\left[\mathrm{Ca}^{2+}\right]_{b}^{2}+K_{p}^{2}}
$$

respectively, where $\left[\mathrm{Ca}^{2+}\right]_{b}$ is a constant (Table I).

As shown in the bifurcation diagram (Fig. 3), the system admits a single low $\left[\mathrm{Ca}^{2+}\right]$ stable steady state for small values of $\Sigma_{\mathrm{MF}}$. These parametric conditions coincide with those for which puffs were observed in the aforementioned stochastic simulations (for which $\Sigma_{\mathrm{MF}}=$ $\left.500 \times 0.01=5 \mu \mathrm{M} \mathrm{s}^{-1}\right)$. For large values of $\Sigma_{\mathrm{MF}}$ ( $>75 \mu \mathrm{M} \mathrm{s}^{-1}$ ), one finds a stable steady state with high levels of $\left[\mathrm{Ca}^{2+}\right]$, in agreement with experiments [2]. For intermediate values of $\Sigma_{\mathrm{MF}}$, the mean-field approach predicts the development of oscillations in a domain bounded by Hopf bifurcation points HB1 and HB2 (Fig. 3). The period of these oscillations is in the range of the mean ISIs reported in the literature [11]. This 


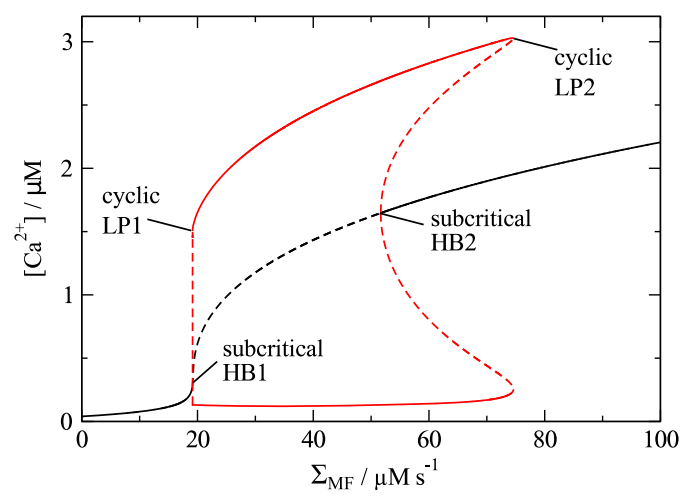

FIG. 3. Bifurcation diagram showing the stationary $\left[\mathrm{Ca}^{2+}\right]$ (in black) and the maximal and minimal $\left[\mathrm{Ca}^{2+}\right]$ reached during oscillations (in red) for $k_{c o}=20 \mu \mathrm{M}^{-1} \mathrm{~s}^{-1}, v_{p}=0.9 \mu \mathrm{M} \mathrm{s}^{-1}$, and the other parameter values given in Table I. Stable and unstable branches are in plain and dashed lines, respectively. HB and LP stand for Hopf bifurcation and fold, respectively.

suggests that the $\mathrm{Ca}^{2+}$ spikes observed experimentally could correspond to oscillations of the limit-cycle type, which would emerge whenever $\bar{\rho}_{c}$ or $\Sigma$ are increased.

We then performed stochastic simulations to track the possible emergence of spikes in multicluster systems. Starting from the puff regime, we ran stochastic simulations for gradually increasing values of $\bar{\rho}_{c}$. By doing so, we enhanced the coupling strength between the clusters and increased $\Sigma_{\mathrm{MF}}$ to get closer to the parametric conditions leading to oscillations in deterministic simulations. Clusters of $0.5 \times 0.5 \mu \mathrm{m}^{2}$ were randomly distributed in a $5 \times 5 \mu \mathrm{m}^{2}$ system according to $\bar{\rho}_{c}$, with the same other parametric conditions as in the puffs' simulations. In this case, $\left[\mathrm{Ca}^{2+}\right]$ was averaged over the whole system. For $\Sigma_{\mathrm{MF}}<25 \mu \mathrm{M} \mathrm{s}^{-1}$, the average $\left[\mathrm{Ca}^{2+}\right]$ remains close to the basal state and small peaks, corresponding to puffs at the local scale, are observed. Beyond this threshold, the global dynamics transforms into global spikes with an amplitude of at least $1 \mu \mathrm{M}$, which is close to the value predicted by the mean-field model. A typical sequence of such spikes is shown in Fig. 2(b). Similar to what is observed in an experimental time series [5], rapid puff activity is visible between spikes. The $\sigma_{\mathrm{ISI}}-T_{\text {av }}$ diagram shown in Fig. 4(a), where each point corresponds to a different realization performed with the same set of parameter values, suggests that a linear relation exists between the standard deviation and the average ISI of the $\mathrm{Ca}^{2+}$ spikes. Additionally, the line and the $x$ axis intersect at a positive value. These statistical properties of the simulated spikes at $\Sigma_{\mathrm{MF}}>$ HB1 are consistent with those of experimental signals $[11,21]$. In a small range of values of $\Sigma_{\mathrm{MF}}$ below HB1 $\left(15.5 \mu \mathrm{M} \mathrm{s}^{-1} \leq \Sigma_{\mathrm{MF}}\right)$, spiking can also occur but with a significantly higher coefficient of variation $(C V)$ (see Fig. S1 in Supplemental Material [26]). This agrees with the $C V$ measured in hepatocytes stimulated at subthreshold concentrations of hormones [28].

We next investigated the $\mathrm{Ca}^{2+}$ spiking dynamics for other parametric conditions leading to oscillations in the mean-field approximation. Spike trains were first obtained with random values of $\Sigma_{\mathrm{MF}}$ (with $\mathrm{HB} 1 \leq \Sigma_{\mathrm{MF}} \leq \mathrm{HB} 2$ ) to simulate various $\mathrm{IP}_{3}$ concentrations. As reported in [21], the corresponding $\sigma_{\mathrm{ISI}}-T_{\mathrm{av}}$ points are positioned along the same straight line (see Fig. S2 in the Supplemental Material [26]). Besides, the slope $\alpha$ is only slightly affected by variations of parameters like $k_{c o}$ [Fig. 4(b)] and $v_{p}$ [Fig. 4(c)], which is consistent with experimental results showing the robustness of $\alpha$ against various pharmacological perturbations, notably inhibiting the SERCA pumps [11]. The slight increment of the slope when $k_{c o}$ is decreased [Figs. 4(a) and 4(b)] can be attributed to a decrease in the ratio between the rates of "firing" and of recovery from inhibition [29].

To check that the $\mathrm{Ca}^{2+}$ spikes analyzed in Fig. 4 correspond to the noise-perturbed limit-cycle oscillations predicted by the mean-field analysis, shown in Fig. 3, we
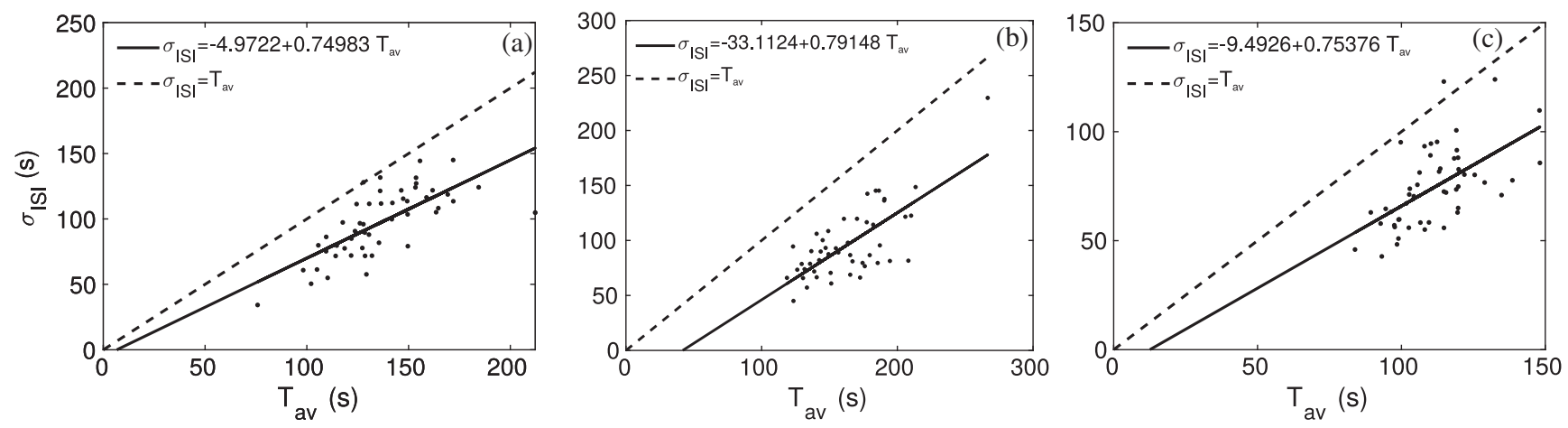

FIG. 4. $\sigma_{\text {ISI }}-T_{\text {av }}$ plot obtained by numerical simulations with $\Sigma=500 \mu \mathrm{M} \mathrm{s}^{-1}, \bar{\rho}_{c}=0.1$ (average intercluster distance of $1 \mu \mathrm{m}$ ), and (a) $k_{c o}=20 \mu \mathrm{M}^{-1} \mathrm{~s}^{-1}$ and $v_{p}=0.9 \mu \mathrm{M} \mathrm{s}^{-1}$, (b) $k_{c o}=5 \mu \mathrm{M}^{-1} \mathrm{~s}^{-1}$ and $v_{p}=0.9 \mu \mathrm{M} \mathrm{s}^{-1}$, and (c) $k_{c o}=20 \mu \mathrm{M}^{-1} \mathrm{~s}^{-1}$ and $v_{p}=0.7 \mu \mathrm{M} \mathrm{s}^{-1}$. The other parametric values are given in Table I. Each dot corresponds to a given spike sequence of at least ten spikes and the solid line is the linear fit through these points. $T_{\min }$ is equal to (a) $6.6 \mathrm{~s}$, (b) $41.8 \mathrm{~s}$, and (c) $12.59 \mathrm{~s}$. The dashed line corresponds to the case $\alpha=1$. 


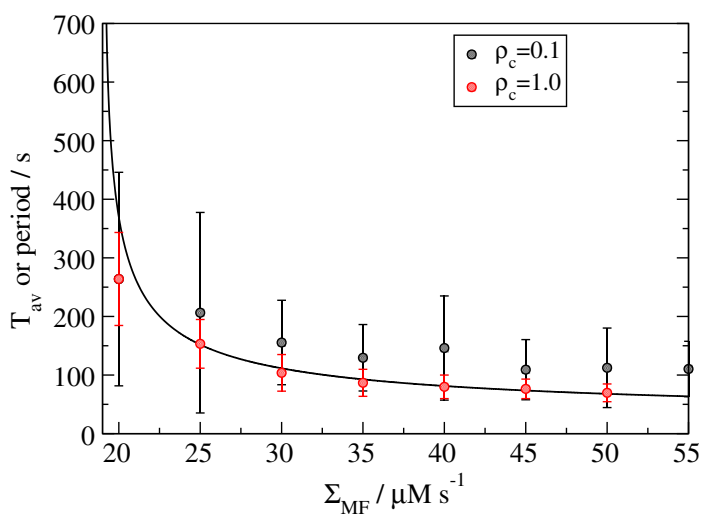

FIG. 5. Comparison between the deterministic period (plain line) and the average ISI (dots) as a function of $\Sigma_{\mathrm{MF}}$ in the range between $\mathrm{HB} 1$ and $\mathrm{HB} 2$. Black and red dots correspond to $\rho_{c}=0.1$ and $\rho_{c}=1.0$, respectively. Error bars indicate variations of $\pm \sigma_{\text {ISI }}$.

compared the deterministic periods and the ISI distributions in the stochastic simulations for the whole range of $\Sigma_{\mathrm{MF}}$ values between HB1 and HB2 (Fig. 5). For the cluster density used in Figs. 2(b) and $4\left(\bar{\rho}_{c}=0.1\right)$, the deterministic period indeed lies in the distribution of the ISI. As expected from the conditions of validity of the mean-field description, the agreement between the stochastic and deterministic simulations improves as the effective cluster density $\bar{\rho}_{c}$ increases (Fig. 5).

In conclusion, we showed through this phenomenological model for spike nucleation that the deterministic counterpart of spiking dynamics can be oscillatory. The onset of stochastic spikes or mean-field oscillations is controlled by the parameters affecting the spatial coupling between the clusters, which is in agreement with experimental data. Moreover, the fully stochastic version of this model generates puff and spike sequences with realistic timescales and statistical properties. The results presented in this Letter thus highlight the compatibility of mean-field limit-cycle behavior with stochastic nucleation mechanisms, which tends to reconcile the current stochastic and deterministic modeling approaches of $\mathrm{Ca}^{2+}$ dynamics.

G. D. and V. V. acknowledge the financial support of the F. R. S.-FNRS. S. P. D. acknowledges the support of UBA (UBACyT 20020170100482BA), ANPCyT (PICT 20153824), and of the Université libre de Bruxelles where this work started during a research stay of hers on a "Chaire Internationale" provided by Université libre de Bruxelles.

*vvoorslu@ulb.ac.be

[1] M. J. Berridge, P. Lipp, and M. D. Bootman, The versatility and universality of calcium signalling, Nat. Rev. Mol. Cell Biol. 1, 11 (2000).

[2] G. Dupont, M. Falcke, V. Kirk, and J. Sneyd, Models of Calcium Signalling, Interdisciplinary Applied Mathematics
(Springer International Publishing Switzerland, 2016), Vol. 43.

[3] J. Shuai, H. J. Rose, and I. Parker, The number and spatial distribution of $\mathrm{IP}_{3}$ receptors underlying calcium puffs in Xenopus oocytes, Biophys. J. 91, 4033 (2006).

[4] S. M. Wiltgen, I. F. Smith, and I. Parker, Superresolution localization of single functional $\mathrm{IP}_{3} \mathrm{R}$ channels utilizing $\mathrm{Ca}^{+}$ flux as a readout, Biophys. J. 99, 437 (2010).

[5] J. S. Marchant and I. Parker, Role of elementary $\mathrm{Ca}^{+}$puffs in generating repetitive $\mathrm{Ca}^{+}$oscillations, EMBO J. 20, 65 (2001).

[6] J. T. Lock, I. F. Smith, and I. Parker, Comparison of $\mathrm{Ca}^{+}$ puffs evoked by extracellular agonists and photoreleased $\mathrm{IP}_{3}$, Cell Calcium 63, 43 (2017).

[7] A. Skupin, H. Kettenmann, U. Winkler, M. Wartenberg, H. Sauer, S. C. Tovey, C. W. Taylor, and M. Falcke, How does intracellular $\mathrm{Ca}^{+}$oscillate: By chance or by the clock? Biophys. J. 94, 2404 (2008).

[8] K. Thurley, I. F. Smith, S. C. Tovey, C. W. Taylor, I. Parker, and M. Falcke, Timescales of $\mathrm{IP}_{3}$-evoked $\mathrm{Ca}^{+}$spikes emerge from $\mathrm{Ca}^{+}$puffs only at the cellular level, Biophys. J. 101, 2638 (2011).

[9] D. Fraiman, B. Pando, S. Dargan, I. Parker, and S. P. Dawson, Analysis of puff dynamics in oocytes: Interdependence of puff amplitude and interpuff interval, Biophys. J. 90, 3897 (2006).

[10] R. Thul and M. Falcke, Stability of Membrane Bound Reactions, Phys. Rev. Lett. 93, 188103 (2004).

[11] K. Thurley, S. C. Tovey, G. Moenke, V. L. Prince, A. Meena, A. P. Thomas, A. Skupin, C. W. Taylor, and M. Falcke, Reliable encoding of stimulus intensities within random sequences of intracellular $\mathrm{Ca}^{+}$spikes, Sci. Signal. (Online) 7, ra59 (2014).

[12] A. Politi, L. D. Gaspers, A. P. Thomas, and T. Höfer, Models of $\mathrm{IP}_{3}$ and $\mathrm{Ca}^{+}$oscillations: Frequency encoding and identification of underlying feedbacks, Biophys. J. 90, 3120 (2006).

[13] M. Falcke, M. Moein, A. Tilūnaite, R. Thul, and A. Skupin, On the phase space structure of $\mathrm{IP}_{3}$ induced $\mathrm{Ca}^{+}$signalling and concepts for predictive modeling, Chaos 28, 045115 (2018).

[14] J. Sneyd, J. M. Han, L. Wang, J. Chen, X. Yang, A. Tanimura, M. J. Sanderson, V. Kirk, and D. I. Yule, On the dynamical structure of calcium oscillations, Proc. Natl. Acad. Sci. U.S.A. 114, 1456 (2017).

[15] A. Skupin and M. Falcke, From puffs to global $\mathrm{Ca}^{+}$signals: How molecular properties shape global signals, Chaos 19, 037111 (2009).

[16] A. Tilūnaitè, W. Croft, N. Russell, T. C. Bellamy, and R. Thul, A Bayesian approach to modelling heterogeneous calcium responses in cell populations, PLoS Comput. Biol. 13, e1005794 (2017).

[17] A. E. Bugrim, A. M. Zhabotinsky, and I. R. Epstein, Calcium waves in a model with a random spatially discrete distribution of $\mathrm{Ca}^{+}$release sites, Biophys. J. 73, 2897 (1997).

[18] P. Jung, D. Swaminathan, and A. Ullah, Calcium spikes: Chance or necessity? Chem. Phys. 375, 625 (2010).

[19] S. Swillens, G. Dupont, L. Combettes, and P. Champeil, From calcium blips to calcium puffs: Theoretical analysis of the requirements for interchannel communication, Proc. Natl. Acad. Sci. U.S.A. 96, 13750 (1999). 
[20] L. Bruno, G. Solovey, A. C. Ventura, S. Dargan, and S. P. Dawson, Quantifying calcium fluxes underlying calcium puffs in Xenopus laevis oocytes, Cell Calcium 47, 273 (2010).

[21] A. Skupin, H. Kettenmann, and M. Falcke, Calcium signals driven by single channel noise, PLoS Comput. Biol. 6, e1000870 (2010).

[22] A. Calabrese, D. Fraiman, D. Zysman, and S. Ponce Dawson, Stochastic fire-diffuse-fire model with realistic cluster dynamics, Phys. Rev. E 82, 031910 (2010).

[23] M. D. Bootman, M. J. Berridge, and P. Lipp, Cooking with calcium: The recipes for composing global signals from elementary events, Cell 91, 367 (1997).

[24] L. F. Lopez and S. Ponce Dawson, Luminal $\mathrm{Ca}^{+}$dynamics during $\mathrm{IP}_{3} \mathrm{R}$ mediated signals, Phys. Biol. 13, 036006 (2016).

[25] D. T. Gillespie, Stochastic simulation of chemical kinetics, Annu. Rev. Phys. Chem. 58, 35 (2007).
[26] See Supplemental Material at http://link.aps.org/ supplemental/10.1103/PhysRevLett.122.088101 for more information on the stochastic algorithm, derivation of the deterministic evolution equations, evolution of the coefficient of variation with respect to $\Sigma_{\mathrm{MF}}$ and statistics of $\sigma_{\text {ISI }}-T_{\text {avg }}$ relation for varying $\Sigma_{\mathrm{MF}}$.

[27] D. Thomas, P. Lipp, M. J. Berridge, and M. D. Bootman, Hormone-evoked elementary $\mathrm{Ca}^{+}$signals are not stereotypic, but reflect activation of different size channel clusters and variable recruitment of channels within a cluster, J. Biol. Chem. 273, 27130 (1998).

[28] G. Dupont, A. Abou-Lovergne, and L. Combettes, Stochastic Aspects of Oscillatory $\mathrm{Ca}^{+}$Dynamics in Hepatocytes, Biophys. J. 95, 2193 (2008).

[29] A. Givré and S. Ponce Dawson, Information content in stochastic pulse sequences of intracellular messengers, Front. Phys. 6, 74 (2018). 